\title{
The research on the fluorescence image of the hydraulic leakage acquisition and analysis
}

\author{
Yufeng Zhang ${ }^{\mathrm{a}}$, Xi Zhang and Minrui Li \\ Wuhan Mechanical Technology college, Wuhan 430075, China \\ shujian59@163.com
}

Keywords: Computer; Hydraulic leakage; Fluorescence image; Detection; Characteristic parameter

\begin{abstract}
This paper introduced the principle of the fluorescence image of the hydraulic leakage acquisition and analysis system. The function and structure of system and the composition diagram of automatic detection hardware had been analyzed. The thresholding and semithresholding on the fluorescence image of the hydraulic leakage by the maximum variance algorithm, the algorithm for edge detection and acquisition the characteristic of fluorescence image were expounded. The program flow chart, the fluorescence image of the hydraulic leakage and the statistics result were provided.
\end{abstract}

\section{Introduction}

The farm machinery was mostly controlled with the hydraulic. The hydraulic fluid seeped was a common phenomenon in the hydraulic system. The leakage of the hydraulic system would pollute the environment, and seriously influenced normal operation. The leak detection had become the important topic in research and practice. The hydraulic fluid seeped of the equipment was the matter which the modernized enterprise must solved, and the examination was also the essential critical process in the equipment production. At present, the methods of detection hydraulic fluid leakage mainly included those, such as visual detection, soapy water froth, talcum powder, ion detector, compressed air [1] and so on. But those methods had various defects and limitations, especially when detected tiny leakage. Not only the working efficiency was low, the labor intensity was heavy, and very easy to cause the omission, the detection mistake. The fluorescence detection technology had provided the new way for detection leakage. The fluorescence leak detection - used ultraviolet/blue light detector lamp and the fluorescence powder, could fast, simple, found the smallest leakage accurately. the fluorescence image of the hydraulic leakage acquisition and analysis system was based on the computer vision hydraulic fluid leakage automatic detection system, had provided the new method for the hydraulic fluid leak detection.

\section{Structure}

It was mainly composed of three parts: Image acquisition, image processing, analysis and control. The array CCD was the image acquisition senso, real-time induction hydraulic fluid leakage pictorial information. USB was the communication interface, realizes the high speed data transmission. The image processing choice threshold value carried on thresholding processing to the image, gave the thresholding image, underlines leakage image [2]. Carried on the computation, the output to the hydraulic fluid leakage characteristic parameter, gave the warning and so on real-time control.

\section{Hardware}

The hardware diagram of the fluorescence image of the hydraulic leakage acquisition and analysis system was shown in Figure 1. DSP (TMS320F2812) GPIOA produced the time sequence pulse as the I/O output interface which CCD needed; Under the time sequence pulse drive, outputs various elements photosensitive signal SOUT, after transported puts the AD8031 processing, outputs 
TMS320F2812 the AIN0 simulation quantity input end; CY7C68013 took and the superior CP machine serial communication interface.

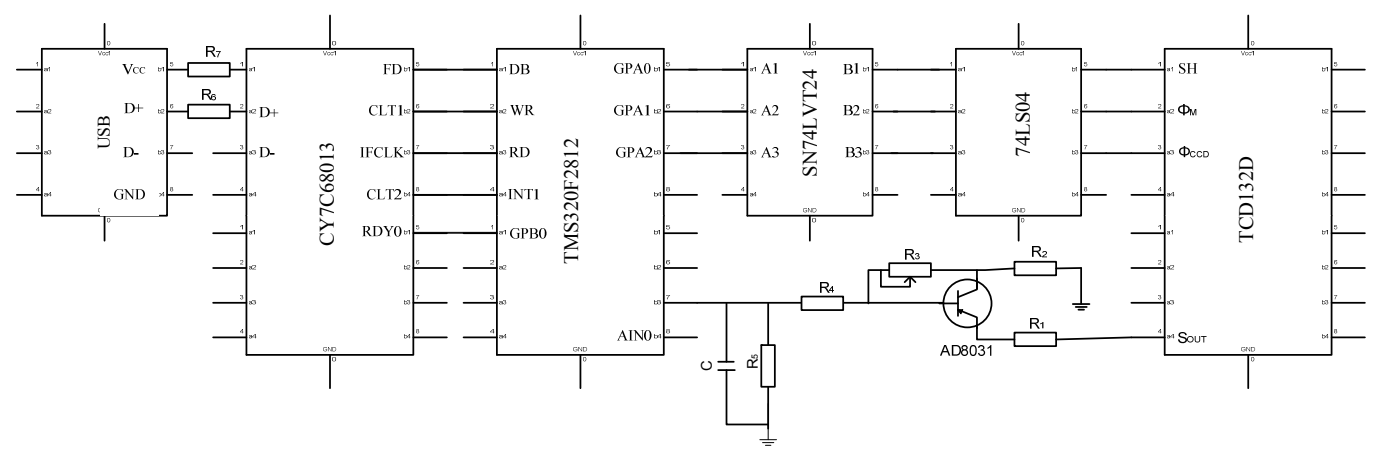

Fig1. The hardware diagram of the fluorescence image

Image sensor. The structure of image sensor was shown in Figure 2. The shell of CCD and LED was made by the aluminum alloy, with black anodic oxidation surface treatment. The CCD placed in the center of the image sensor. LED (white light) and LED (ultraviolet ray) installed alternately in the shell, the shell of CCD and the LED shell contact face spread the black waterproof adhesive.

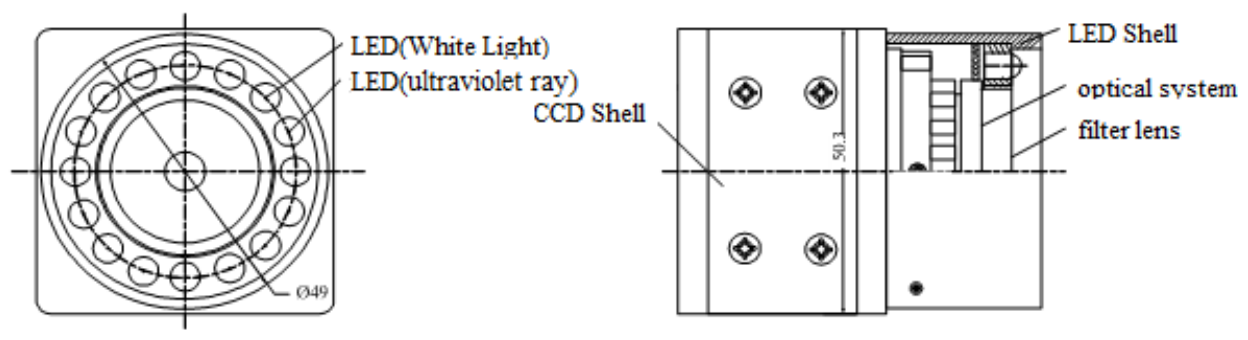

Fig2. The structure diagram of image sensor

CCD was a integration sensor with spotlight phonograph trades, the charge storage, the charge transfer, which had the high resolution, the simple structure, the simple output signal processing. So it obtained the widespread application. According to accuracy requirement of online examination, selected the TCD132D component, its element size was $14 \mu \mathrm{m}, 1024$ effective photosensitive element, the driving frequency was highest may reach $4 \mathrm{MHz}$; Could satisfy the sampling theorem and the measuring accuracy request.

Processor. DSP was one kind had the special structure microprocessor, the interior used the procedure and the data separated Harvard structure, had the special hardware multiplier, widely used the stream line operation, could use for to realize each kind of data acquisition and the digital signal processing fast.

According to fluorescence detection hydraulic fluid leakage image information acquisition as well as the CCD driving frequency's request, selected high speed DSP-TMS320F2812. It had 16 lines 12B the highest gathering frequency $25 \mathrm{MHz} \mathrm{A} / \mathrm{D}$ channels, the interior storage space for $128 \mathrm{k} \times 16 \mathrm{~B}$ the exterior expansion space for $1 \mathrm{M} \times 16 \mathrm{~B}$; the processing speed was $150 \mathrm{MB} / \mathrm{s}$.

USB serial bus. USB was one kind of universal serial bus, the speed was quickly the USB technology prominent characteristic, the connection maximum transmissibility might reach $480 \mathrm{MB} / \mathrm{s}$, was general serial port 100 times, could realize the data speedy transmission. CY7C68013 was one of based on USB2.0 agreement equipment end used many chips. Had the parallel main line and the partial DMA through-put capacity high speed connection component. Might with any exterior micro controller/microprocessor realize the high speed parallel interface $(25 \mathrm{MB} / \mathrm{s})$. 


\section{Leakage image information acquisition and analysis}

The threshold value of Biggest variance territory value method. The principle of the image measurement system was through processing was measured that the object image the edge obtains the object the geometric parameter, because the peripheral point was located in generally the image the grey level change fierce position, namely the grey level derivative was big or the enormous place, therefore marginal check's method was generally first through the gradient or the differential algorithm peaking picture edge, after then used some kind of template and the peaked image carries on the convolution to obtain the edge contour image. The marginal check had many algorithms, like the gradient operator, the Prewitt operator, the Marr operator, the Sohle operator, the Kirsch operator and so on, in which algorithm's fit and unfit quality were deciding the edge contour extraction precision height. In this measurement system, because in the image was measured that the damage spot with has not damaged the spot to ask the contrast gradient was big, the image was relatively quite simple, therefore we also used one on the other hand quite simple algorithm.

The image generally included the object, the background and the noise usually, must withdraw the object from the multiple-valued digital image, must first through the filtering algorithm elimination noise spot, then establish some territory value $\theta$, with $\theta$ the image data division was two parts: Was bigger than $\theta$ the picture element group and Was smaller than $\theta$ picture element group. The computation territory value's algorithm had the biggest variance territory value law generally, smallest variance territory value law, difference histogram law and Laplace methods and so on histogram law [3]. We had chosen the biggest variance territory value law, it was based on the least squares method inferential reasoning, but results. Realizes the method to be as follows specifically:

Supposes an image the grey level respectively was the $0 \sim \mathrm{m}$ level, the grey level was $\mathrm{i}$ image element ni, then the total image element is: $N=\sum_{i=0}^{m} n_{i}$.

The probability for gray scales is $p_{i}=n_{i} / N$. Then the gray scales are divided into two groups $\mathrm{C}_{0}=\{0 \sim \mathrm{k}\}$ and $\mathrm{C}_{1}=\{\mathrm{k}+1 \sim \mathrm{m}\}$, and the probability for each group is respectively:

Probability of $\mathrm{C}_{0}: w_{0}=\sum_{i=0}^{k} p_{i}=w(k)$; probability of $\mathrm{C}_{1}: w_{1}=\sum_{i=0+1}^{m} p_{i}=1-w(k)$.

From the two equations the mean of $\mathrm{C}_{0}$ :

$$
u_{0}=\sum_{i=0}^{k}\left(i p_{i} / w_{0}\right)=u(k) / w(k)
$$

The mean of $\mathrm{C}_{1}$ :

$$
u_{1}=\sum_{i=0+1}^{m}\left(i p_{i} / w_{1}\right)=[u-u(k)] /[1-w(k)]
$$

Here $u_{0}=\sum_{i=0}^{k} p_{i}$ is the mean grayscale of whole image, $u(k)=\sum_{i=0}^{k} p_{i}$ is the mean grayscale when the threshold is $\mathrm{k}$, so the mean grayscale of all samples: $\mathrm{u}=\mathrm{w} 0 \mathrm{u} 0+\mathrm{w} 1 \mathrm{u} 1$. The variance of the two groups can be obtained by the equation below:

$$
\begin{aligned}
& \sigma_{2}(k)=w_{0}\left(u-u_{0}\right)^{2}+w_{1}\left(u_{1}-u\right)^{2}=w_{0} w_{1}\left(u_{1}-u_{0}\right)^{2} \\
& =[u w(k)-u(k)]^{2} /\left\{[w(k)[1-w(k)]\}^{2}\right.
\end{aligned}
$$

Changed the $\mathrm{k}$ value from $0 \sim \mathrm{m}$, The computation above equation was $\mathrm{c} 0$ 。 Time the biggest namely $\max _{z}(k)_{\text {时 }} \mathrm{k}$ value, this time $\mathrm{k}$ is the territory value which asks $\theta$.

Half thresholding processing. According to territory value which calculates $\theta$, Would be bigger than was equal to $\theta$ the grey level supposes was 255 . Was smaller than $\theta$ the grey level supposes was 0 。 Supposes the input image was $f(x, y)$, the output image $f(x, y)$, then: 


$$
f(x, y)=225 f(x, y) \geq \theta, 0 f(x, y) \leq \theta
$$

Realizes the method was specifically with reads each spot in Visual $\mathrm{C}++4.1$ by the row the image element value, was bigger than the grey level $\theta$ the spot to set is 255 , was smaller than $\theta$ the spot sets is 0 realizes.

After image half thresholding processing, retained a leakage image only, but expresses the normal background as white.

Marginal check and leakage characteristic parameter seeking. The marginal check with gradient operator method seeking, regarding half thresholding image function $\mathrm{f} \theta(\mathrm{x}, \mathrm{y})$, was selecting $(\mathrm{x}, \mathrm{y})$ gradient was a vector, the definition was:

$$
\Delta f_{\theta}(x, y)=\left[\frac{a f_{\theta}}{a x} \frac{a f_{\theta}}{a y}\right]^{\theta}
$$

Gradient direction in the function $f_{\theta}(x, y)$ maximum rate of change, gradient scope $\left|\Delta f_{\theta}(x, y)\right|$ may figure out by (6) the formula:

$$
\left|\Delta f_{\theta}(x, y)\right|=\left[\left(\frac{a f_{\theta}}{a x}\right)^{2}+\left(\frac{a f_{\theta}}{a y}\right)^{2}\right]^{1 / 2}
$$

The gradient value was the quantity which was away from in its maximum rate of change direction's unit increases. (6) the formula may rewrite was:

$$
\left|\Delta f_{\theta}(x, y)\right|=\left\{\left[f_{\theta}(x, y)-f_{\theta}(x+1)\right]^{2}+\left[f_{\theta}(x, y)-f_{\theta}(x, y+1)\right]^{2}\right\}^{1 / 2}
$$

Using the level vertical method of difference (6) the formula simplification was:

$$
\left|\Delta f_{\theta}(x, y)\right|=\left|f_{\theta}(x, y)-f_{\theta}(x+1, y)\right|+\left|f_{\theta}(x, y)-f_{\theta}(x, y+1)\right|
$$

May know by the gradient computation, in the image, the gradation change big border region its gradient value was big, was small in the gradation change gentle region its gradient value, but in the gradation even region its gradient value was zero.

Marginal check arithmetic was take row column, leakage edge clearly as principle [4]. The good marginal check obtained a leakage width. The row marginal check obtained a leakage length; According to the leakage edge clarity, obtained a leakage area. The leakage characteristic parameter (extreme breadth, the greatest length, area took likely element as fundamental unit, converts for actual area).

\section{Conclusions}

The hydraulic fluid leakage image was gathered to the computer, to the picture edge detection, the threshold value division and half thresholding processing, the seeking hydraulic fluid leakage image characteristic, could examine the hydraulic fluid leaking point effectively, the computation leakage characteristic parameter and the position, could satisfy the hydraulic fluid leakage the detection request; Carried on the examination using that system to the hydraulic fluid leakage, had the automaticity to be high, the examination time was short, time-saving, reduced effort, could enhance the accuracy and the objectivity.

\section{References}

[1]X.L. Wang, X. Xu and W. Wang. Natural Gas and Petroleum, Vol. 25 (2007) No. 3, p. 21-13. (In Chinese)

[2]X.Y. Cheng and J.M. Zhang. Journad of Shantou University(Natural Science), Vol. 21 (2006) No. 1, p. 65-69. (In Chinese)

[3]A.G. Lin and B.Y. Sun. Aquatic Product Journal of Fisheries of China, Vol. 30 (2006) No. 3, p. 397-403. (In Chinese) 
[4]W. Wang, Y.G. Deng and W.X. Wei, System Simulation Academic Journal, Vol. 18 (2006) No. 1, p. 425-429. (In Chinese) 